\section{Diagnóstico de neumatosis gástrica en pacientes pediátricos. Reporte de serie de casos}

\author{
Manuel Gil-Vargas, ${ }^{1}$ José Luis Balanzar-Depraect, ${ }^{1}$ Guillermo Victoria- \\ Morales, ${ }^{1}$ Mariana Lee Miguel-Sardaneta, ${ }^{2}$ Héctor Alonso López-Santos, ${ }^{3}$ \\ Angélica Ramírez-Servín, ${ }^{4}$ Michelle Rosas-Téllez ${ }^{4}$
}

\begin{abstract}
Resumen
La neumatosis gástrica es la presencia de aire en la pared del estómago. Es un signo radiológico extremadamente raro en pediatría causado por lesiones microscópicas de la mucosa gástrica. Se presentan cuatro casos de pacientes pediátricos con diagnóstico de neumatosis gástrica atendidos entre el 1 de enero de 2010 y el 31 de diciembre de 2015. Los pacientes presentaron similitudes en sus síntomas como vómito, fiebre y dolor abdominal. El caso dos tuvo antecedente personal patológico de síndrome de Down y el caso cuatro antecedente de laparotomía exploradora por torsión axial del estómago. Se sometieron a cirugía todos los casos con diagnóstico preoperatorio de neumatosis gástrica. Los casos 1 y 4 fueron objeto de segunda intervención quirúrgica por obstrucción intestinal. Se analizan 4 de 5 diagnósticos debido a que un caso clínico no contó con diagnóstico operatorio pues el paciente causó alta voluntaria. La neumatosis gástrica es un signo radiológico que, de acuerdo con sus características, orienta a sospechar una obstrucción gastroduodenal. Así mismo, se han encontrado diversos casos de neumatosis gástrica en pacientes con síndrome de Down.
\end{abstract}

PALABRAS CLAVE: neumatosis gástrica, gastritis enfisematosa, neumatosis quística, enfisema gástrico.

\section{Diagnosis of gastric pneumatosis in pediatric patients. Case report series.}

Manuel Gil-Vargas, ${ }^{1}$ José Luis Balanzar-Depraect, ${ }^{1}$ Guillermo VictoriaMorales, ${ }^{1}$ Mariana Lee Miguel-Sardaneta, ${ }^{2}$ Héctor Alonso López-Santos, ${ }^{3}$ Angélica Ramírez-Servín, ${ }^{4}$ Michelle Rosas-Téllez ${ }^{4}$

\section{Abstract}

Gastric pneumatosis is the presence of air in the stomach wall. It is a radiological sign extremely rare in the pediatric population, caused by microscopic injuries in the gastric mucosa. We described 4 clinical cases of gastric pneumatosis in pediatric patients during January $1^{\text {st }}$, 2010 to December 31 1 st 2015.

\footnotetext{
${ }^{1}$ Cirujano pediatra, Servicios de Salud del Estado de Puebla, Puebla, México.

${ }_{2}^{2}$ Pediatra, Servicios de Salud del Estado de Puebla, Puebla, México.

${ }^{3}$ Residente de Pediatría, Servicios de Salud del Estado de Puebla, Puebla, México.

${ }^{4}$ Médico pasante en Servicio Social, Universidad Popular Autónoma del Estado de Puebla, Puebla, México.
}

Recibido: 5 de septiembre 2016

Aceptado: 25 de abril 2017

\section{Correspondencia \\ Manuel Gil Vargas \\ gilvm@yahoo.com}

\section{Este artículo debe citarse como} Gil-Vargas M, Balanzar-Depraect JL, Victoria-Morales G, Miguel-Sardaneta ML, López-Santos HA, RamírezServín $A$ et al. Diagnóstico de neumatosis gástrica en pacientes pediátricos. Reporte de serie de casos. Acta Pediatr Mex. 2018;39(2):179-184. 
The 4 patients presented similarities in the symptomatology, such as vomiting, fever and abdominal pain. Cases 2 presented as a personal pathological antecedent Down syndrome, and the case 4 presented history of exploratory laparotomy caused by axial torsion of the stomach. Cases 1 and 4 underwent a second surgical intervention due to intestinal obstruction. 1 patient was a voluntary discharged.

Gastric pneumatosis is a radiological sign that lead us to suspect a gastroduodenal obstruction. Several cases of gastric pneumatosis have been found in patients with Down syndrome.

KEYWORDS: gastric pneumatosis; emphysematous gastritis; quistic neumatosis; gastric emphysema
Correspondence

Manuel Gil Vargas

gilvm@yahoo.com

\section{INTRODUCCIÓN}

La neumatosis gástrica es un signo radiológico que representa la infiltración de gas dentro de la pared gástrica, de etiología infecciosa (entercocolitis necrosante) o no infecciosa, como resultado de una obstrucción gastroduodenal. ${ }^{1}$ La neumatosis gástrica se considera un signo radiológico extremadamente raro en la infancia, se presenta con mayor incidencia en recién nacidos prematuros. ${ }^{2}$

Esta afección fue inicialmente descrita por Duo Vernoi, en 1730, y posteriormente estudiada por Fraenkel en 1987.3

Se presenta una serie de cuatro casos clínicos de pacientes diagnosticados con neumatosis gástrica, en nuestra institución, con el objetivo de aportar información a la sociedad pediátrica sobre dicho hallazgo radiológico. La incidencia reportada a nivel internacional es muy baja.

\section{PRESENTACIÓN DE CASOS CLÍNICOS}

Se presentan cuatro casos con diagnóstico radiológico de neumatosis gástrica durante el periodo del 1 de enero del 2010 al 31 de diciembre del 2015.

\section{Caso 1}

Niña de 6 meses de edad que ingresó al servicio de urgencias por vómito de contenido gástrico y fiebre. Como antecedente personal patológico se informó infección de vías urinarias de repetición. La paciente ingresó a quirófano, a laparotomía exploradora, con diagnóstico de neumatosis gástrica; se encontró neumatosis en ambas curvaturas gástricas y bazo accesorio. Su evolución no fue favorable debido a un bloqueo intestinal parcial localizado por una serie esofagogastrodueodenal donde se observó el signo de doble burbuja. Se decidió un segundo abordaje quirúrgico en el que se localizó la membrana duodenal en la segunda porción del duodeno, liberándose la obstrucción duodenal.

\section{Caso 2}

Niña de 2 años que ingresó al servicio por dolor abdominal. Como antecedentes tenía: síndrome de Down, comunicación interauricular y estreñimiento crónico idiopático. Se decidió realizar laparotomía exploradora con el diagnóstico preoperatorio de aire libre subdiafragmático y neumatosis gástrica. Se localizó un quiste dependiente del omento mayor, bandas de Ladd, malrotación intestinal y divertículo de Meckel. Su evolución posoperatoria fue favorable. 


\section{Caso 3}

Niño de 4 años que ingresó al servicio de urgencias por vómito gástrico y dolor abdominal. Tenía antecedentes de estreñimiento que ameritó hospitalización previa. En la serie esofagogastroduodenal de ingreso se sospechó la existencia de un cuerpo extraño en el estómago. En la panendoscopia se aspiraron $400 \mathrm{~mL}$ de líquido fétido, espeso, con estenosis concéntrica de píloro. Se decidió la intervención quirúrgica por diagnóstico preoperatorio de estenosis concéntrica del píloro y neumatosis gástrica. En la piloroplastia de Mikulickz se encontró hipertrofia de la capa mucosa, engrosamiento de la capa muscular del píloro y neumatosis gástrica. Su evolución fue favorable.

\section{Caso 4}

Niño de 15 años de edad que ingresó por vómito de contenido gástrico y dolor abdominal. Contaba con una cirugía previa dos años antes de su ingreso a urgencias por torsión axial del estómago. El diagnóstico preoperatorio fue neumatosis gástrica con aire libre subdiafragmático, razón por la que se efectuó una laparotomía exploradora en la que se encontraron bandas de Ladd. Se realizó procedimiento de Ladd. Su evolución posquirúrgica no fue favorable debido a que se localizó una obstrucción extrínseca de la tercera porción del duodeno, se realizó liberación de la obstrucción y plastia tipo Mikulickz. Tras la segunda intervención quirúrgica su evolución fue favorable.

En el Cuadro 1 se resumen los cuatro casos clínicos analizados y en el Cuadro 2 los estudios de imagen realizados para el diagnóstico de neumatosis gástrica.

\section{DISCUSIÓN}

La neumatosis gástrica se divide en dos categorías: enfisema gástrico y gastritis enfisematosa.
El mecanismo mediante el cual el aire llega al espesor de la pared del estómago no se conoce con precisión; sin embargo, la teoría más aceptada son las lesiones microscópicas de la mucosa gástrica. ${ }^{2,4} \mathrm{El}$ enfisema gástrico se ha descrito secundario a una obstrucción gastroduodenal como estenosis pilórica, malrotación intestinal, atresia duodenal, membrana duodenal y páncreas anular. ${ }^{5-7}$

La gastritis enfisematosa se origina por isquemia o inflamación de la mucosa gástrica secundaria a enterocolitis necrosante y sepsis. ${ }^{2}$ La inflamación de la mucosa gástrica permite la producción de gas y la proliferación bacteriana. ${ }^{4}$ La clínica permite distinguir entre el enfisema gástrico y la gastritis enfisematosa pero también existen diferentes hallazgos radiológicos que diferencian entre una y otra.

El enfisema gástrico se caracteriza por una línea de aire que rodea a la cámara gástrica. ${ }^{8}$ En la gastritis enfisematosa existen imágenes quísticas de gas o burbujas alrededor del estómago distendido, como las que se observan en la enterocolitis necrosante ${ }^{9-11}$ pero el pronóstico es el que aporta una diferencia marcada. El enfisema gástrico responde al resolverse la causa de la obstrucción gastroduodenal. Se reporta mejoría radiológica a las 4 horas y la resolución entre 16 y 24 horas. ${ }^{4}$ La gastritis enfisematosa, en conjunto con la enterocolitis necrosante, tiene la característica de un pronóstico pobre. ${ }^{2,12}$

El paciente del caso clínico 2 tenía antecedente de síndrome de Down que es un antecedente personal patológico relevante. En la revisión bibliográfica se encontraron dos pacientes con el mismo diagnóstico. Al compararlos se observa que el caso clínico 2 y los dos casos tomados de la bibliografía tuvieron una etiología mecánica (obstrucción), dos casos a nivel de la segunda porción del duodeno y uno a $40 \mathrm{~cm}$ de la vál- 


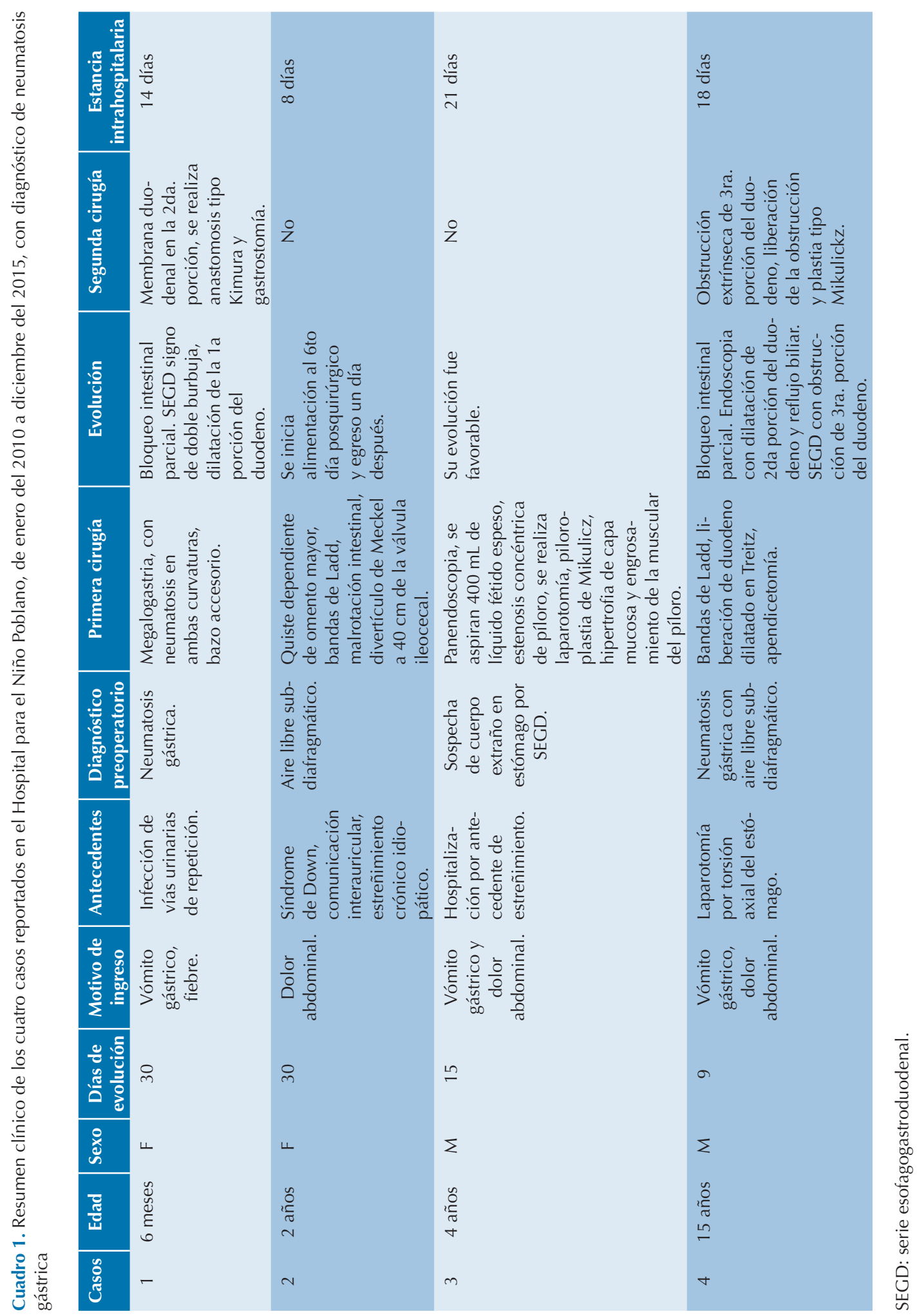




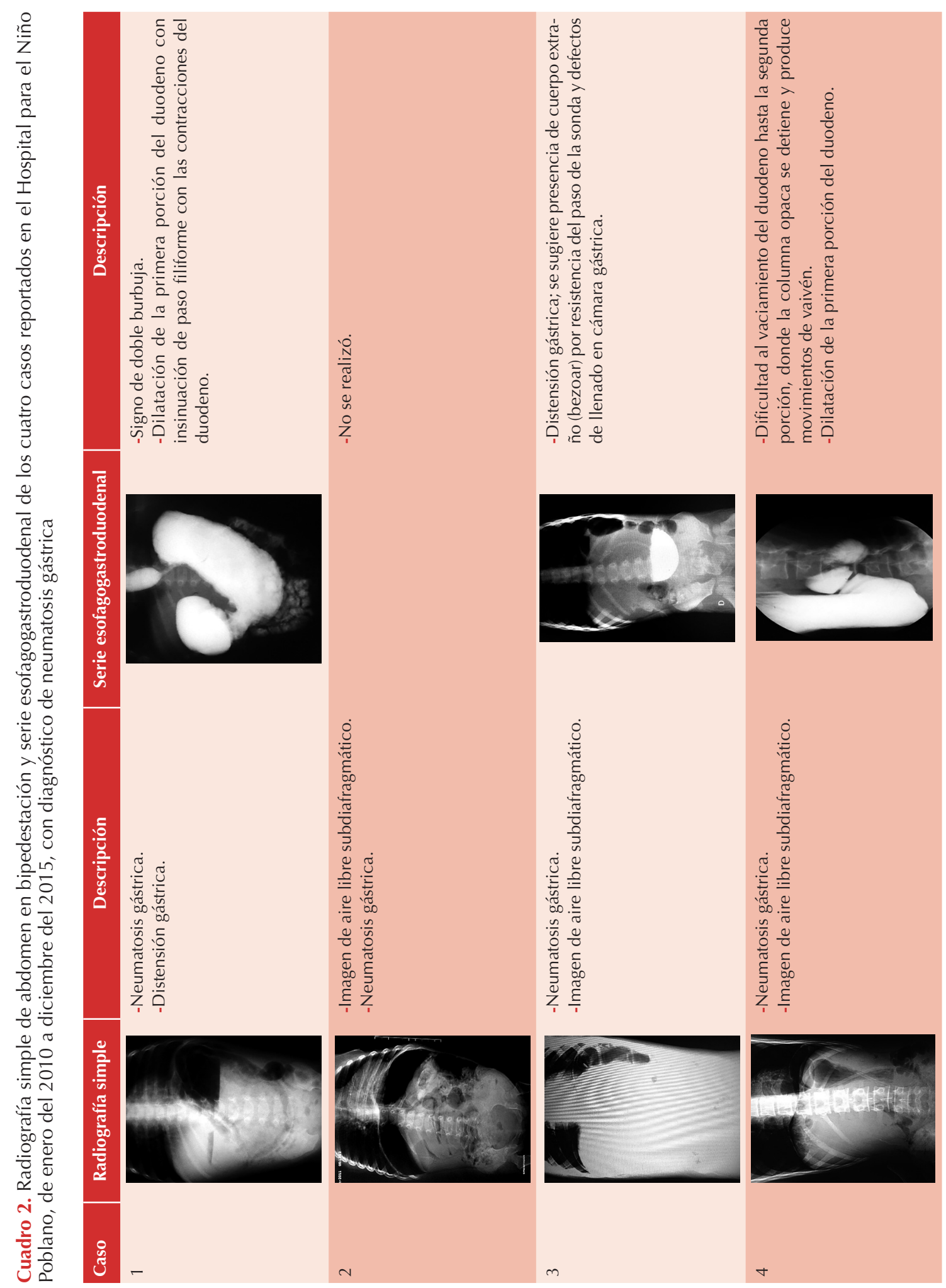


vula ileocecal, lo que nos lleva a puntualizar la importancia de tomar al síndrome de Down como un probable factor de riesgo de obstrucción intestinal y, posteriormente, neumatosis gástrica. ${ }^{13,14}$ Destaca un caso clínico femenino, de 2 años de edad, que tenía como antecedente síndrome de Down pero que fue dado de alta sin procedimiento quirúrgico por alta voluntaria.

\section{CONCLUSIÓN}

La bibliografía de la neumatosis gástrica en pacientes pediátricos no es muy vasta, en parte debido a su baja frecuencia y en parte porque, en sí, no es una enfermedad per se sino un signo radiológico de manifestación de alguna otra enfermedad; en nuestros casos obstrucción gastroduodenal.

La neumatosis gástrica es un signo radiológico que, de acuerdo con sus características, permite sospechar una obstrucción gastroduodenal. Así mismo, se han encontrado diversos casos de neumatosis gástrica en pacientes con síndrome de Down; sin embargo, al existir pocos casos reportados en la bibliografía no se puede concluir, con base en estudios estadísticos, a dicho síndrome como un factor de riesgo.

El reporte de esta serie de casos ayuda a la sociedad pediátrica a tener conocimiento de los hallazgos radiológicos con la finalidad de establecer un diagnóstico oportuno de una probable obstrucción gastroduodenal.

\section{REFERENCIAS}

1. Baeza C, Nájera HM, García LM, Gardida R. Enfisema gástrico intersticial en niños. Rev Gastroenterol Mex. 2000;65(2):74-80.

2. Markel TA, Wanner MR, Billmire DF. Gastric pneumatosis secondary to pyloric stenosis. Journal of Pediatric Surgery. 2013;48:655-657.

3. McKelvie PA, Fink AM. A fatal case emphysematous gastritis and esophagitis. Pathology. 1994;26(4):490-492.

4. D'Cruz, Sherif E. Gastroduodenal emphysema. Journal of Pediatric Surgery. 2008;43(11):2121-2123.

5. Travadi JN, Patole SK, Simmer K. Gastric pneumatosis in neonates: Revisited. Int J Paediatr Child Health. 2003;39(7):560-562.

6. Sebastia C, et al. Portomesenteric vein gas: pathologic mechanisms, CT findings, and prognosis. Radiographics. 2000;20(5):1224-1226

7. Bhargava P, Paris M. Gastric pneumatosis and Portal venous gas: benign findings in hypertrophic pyloric stenosis. Pediatr Radiol. 2009;39(4):413.

8. Duran R, Vatansever U, Aksu B, et al. Gastric pneumatosis intestinalis: an indicator of intestinal perforation in preterm infants with necrotizing enterocolitis?. J Pediatr Gastroeneterol Nutr. 2006;43(4):539-541.

9. Berens SV, Moskowitz H, Mellins HZ. Air within the wall of the stomach. Roentgen manifestations and a new roentgenographic sign. Am J Roentgeno Radium Ther Nucl Med. 1968;103(2):310-313.

10. Arikanoglu Z, Aygen E, Camci C, et al. Pneumatosis cystoides intestinalis: A single center experience. World J Gastroenterol. 2012;18(5):453-457.

11. Morris MS, Gee AC, Cho SD, et al. Management and outcome of pneumatosis intestinalis. Am J Surg. 2008;195(5):679682.

12. Soon MS, Yen HH, Soon A, Lin OS. Endoscopic ultrasonographic appearance of gastric emphysema. World J Gastroenterol. 2005;11(11):1719-1721.

13. Kawano S, Tanaka H, Daimon Y, et al. Gastric pneumatosis associated with duodenal stenosis and malrotation. Pediatr Radiol. 2001;31(9):656-658.

14. Torf CP, Christianson RE. Anomalies in Down Syndrome Individuals in a large population-based registry. Am J of Med Genet. 1998;77(5):431-438. 